\title{
EFECTO BRONCODILATADOR DEL EXTRACTO HIDROALCOHÓLICO DE PROPÓLEO DE Apis mellifera “ABEJA” EN ANILLOS TRAQUEALES AISLADOS DE Cavia porcellus “COBAYOS”
}

\author{
Bronchodilator effect of hydroalcoholic extract of propolis from Apis mellifera "bee" in \\ isolated tracheal rings from Cavia porcellus "guinea pigs"
}

\author{
Mario Carhuapoma ${ }^{1}$, Britt Loayza ${ }^{2}$, Johnny A.Tinco ${ }^{1}$, Sofía López ${ }^{2}$, José Iannacone ${ }^{3}$, Mónica Chávez ${ }^{1}$ \\ ${ }^{1}$ Facultad de Farmacia y Bioquímica, Universidad Nacional Mayor de San Marcos. ${ }^{2}$ Facultad de Ciencias de la Salud, Universidad \\ Nacional de San Cristóbal de Huamanga. ${ }^{3}$ Universidad Nacional Federico Villarreal - Universidad Ricardo Palma.
}

\section{RESUMEN}

El presente trabajo tiene por objetivo determinar el efecto broncodilatador del extracto hidroalcohólico de propóleo de Apis mellifera "abeja" en anillos traqueales aislados de Cavia porcellus "cobayos". El propóleo fue recolectado en el distrito de Chalhuanca, provincia de Aymaraes de la región Apurímac. Se determinaron las características organolépticas y fisicoquímicas del propóleo en bruto, y se realizó el tamizaje fitoquímico del extracto hidroalcohólico. El efecto broncodilatador se determinó mediante el método de Jagdish et al. modificado, utilizando anillos traqueales y haciendo uso de un quimógrafo automatizado,

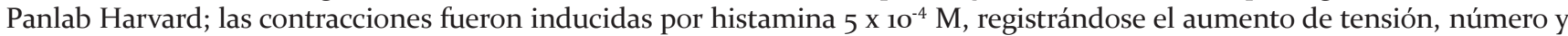
altura de contracciones. Como control positivo se usó salbutamol, el extracto hidroalcohólico se ensayo a las concentraciones de 5, 10 y 15\%. El extracto hidroalcohólico de propóleo al 5\% redujo la tensión a 10,818 g, semejante a lo logrado con salbutamol (10,852 g), obteniéndose mejores resultados al 10 y 15\%, 10,206 y 9,371 g, respectivamente. El número y altura de las contracciones fueron de 21,40 y 9,52 mm con salbutamol; con extracto: 19,20 y 9,24 mm al 5\%; 15,40 y 8,42 $\mathrm{mm}$ al $10 \%$ y 9,80 y $7,30 \mathrm{~mm}$ al $15 \%$. El análisis estadístico mostró diferencias significativas en los diferentes tratamientos ( $p<0,05 \%)$. Se concluye que el extracto hidroalcohólico de propóleo tiene efecto broncodilatador, mostrando mayor efecto al 5\%.

Palabras clave: Propóleo, extracto hidroalcohólico, efecto broncodilatador, Apis mellifera, Cavia porcellus.

\section{SUMMARY}

The present work was carried out in order to determine the effect of bronchodilator of hydroalcoholic extract of propolis from Apis mellifera "bee" in tracheal rings isolated from Cavia porcellus "cobayos". The sample was collected in Chalhuanca district, Aymaraes province_Apurímac region. Were determinated organoleptic and physical-chemical characteristics of raw propolis, and was realized phytochemical screening of hydroalcoholic extract. The bronchodilator effect was determined by the method of Jagdish et al. modified in tracheal rings, using an automated kymograph, Panlab Harvard; contractions were induced by histamine $5 \times 10^{-4} \mathrm{M}$, registering increasing tension, number and height of contractions. It was used as control salbutamol and assessed the hydroalcoholic extract of propolis to concentrations of $5 \%, 10 \%$ and $15 \%$. The hydroalcoholic extract of propolis $5 \%$ reduced tension to $10,818 \mathrm{~g}$ similar to salbutamol (10,852 g), getting better results to $10 \%$ and $15 \%, 10,206 \mathrm{~g}$ and $9,371 \mathrm{~g}$, respectively. The number and height of contractions were 21,40 and 9,52 mm with salbutamol; 19,20 and 9,24 $\mathrm{mm}$ at 5\%; 15,40 and 8,42 $\mathrm{mm}$ at $10 \%$ and finally 9,80 and $7,30 \mathrm{~mm}$ at $15 \%$. Statistical analysis showed significant differences in the different treatments ( $p<0,05 \%)$. Was conclude that the hydroalcoholic extract of propolis has bronchodilator effect, showing greater effect at $5 \%$.

Keywords: Propolis, hydroalcoholic extract, bronchodilator effect, Apis mellifera, Cavia porcellus.

\section{INTRODUCCIÓN}

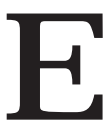
1 propóleo ha sido ampliamente utilizado en la medicina tradicional desde tiempos antiguos, reconocido por las propiedades terapéuticas que se le han atribuido. En la medicina tradicional peruana es usado en afecciones respiratorias -incluyendo asma-, para las que suelen emplearse además resinas, bálsamos y plantas medicinales, en prácticas terapéuticas que se han transmitido de generación en generación, y que han preservado un valioso arsenal de sustancias para la industria farmacéutica.

Según la Organización Mundial de la Salud (OMS), más de 235 millones de personas en el mundo padecen de asma, siendo una de cada 250 muertes, causada por este mal. Las estadísticas también indican que más del 80\% de las muertes por asma ocurren en países de ingresos bajos y medio-bajos ${ }^{(1)}$.

El propóleo es una sustancia resinosa, gomosa y balsámica, obtenido por las abejas obreras de la especie 
Apis mellifera, a partir de los exudados de las cortezas y diversos tejidos de las plantas. Los materiales colectados, son triturados, humedecidos con saliva y secreciones enzimáticas, mezclados con la cera producida por las glándulas céreas y finalmente transportadas por las abejas hacia la colmena, donde cumplen diversas funciones ${ }^{(2)}$.

Es utilizado por la gran variedad de compuestos que presenta, entre estos compuestos fenólicos o polifenoles y flavonoides que han sido reconocidos por su amplio espectro de actividades biológicas, tales como anticancerígenos, analgésicos, antiinflamatorios, inmunomoduladores, antibacterianos, y antioxidantes ${ }^{(3)}$.

Por su propiedad broncodilatadora, es empleado en el tratamientodelasma;porestarazónsedesarrollóelpresente trabajo de investigación que estuvo orientado a conocer los principales metabolitos secundarios responsables de dicha actividad mediante tamizaje fitoquímico, así como corroborar el efecto broncodilatador in vitro utilizando el modelo de anillos traqueales aislados de cobayo, haciendo uso de un quimógrafo automatizado; este equipo tiene un transductor y amplificador con conexión a un computador que permitió registrar la actividad muscular del órgano aislado de manera más precisa. Por estas consideraciones, el objetivo de la presente investigación fue evaluar el efecto broncodilatador del extracto hidroalcohólico de propóleo de Apis mellifera "abeja" en anillos traqueales aislados de Cavia porcellus "cobayos".

\section{MATERIALES Y MÉTODOS}

El presente trabajo de investigación se realizó en los laboratorios de Farmacología y Farmacognosia de la Escuela de Formación Profesional de Farmacia y Bioquímica de la Facultad de Ciencias Biológicas de la Universidad Nacional de San Cristóbal de Huamanga, durante los meses de julio a diciembre de 2013.

Animales de experimentación. Seextrajeron las tráqueas de 15 cobayos Cavia porcellus de l8a misma edad, machos, peso $600+/-50 \mathrm{~g}$, procedentes del Instituto Nacional de Investigación Agraria - Ayacucho, los que fueron acondicionados, con alimentación balanceada y agua a libertad, en el bioterio del Laboratorio de Farmacología del área de Farmacia de la Facultad de Ciencias Biológicas de la Universidad Nacional de San Cristóbal de Huamanga.

Fármaco de referencia. Cien tabletas salbutamol de $4 \mathrm{mg}$, fabricado por Laboratorios Medrock S.A.C., $\mathrm{N}^{\circ}$ de lote 107312.

Solución patrón. Se preparó una solución con 20 $\mathrm{mg} / 10 \mathrm{~mL}$ de agua destilada y se filtró para obtener la solución patrón de salbutamol.
Extracto hidroalcohólico de propóleo. Preparado a concentraciones de 5,10 y $15 \%$, con medio nutricio de Tyrode.

Histamina. Se preparó en agua destilada a una concentración de $5 \times 10^{-4} \mathrm{M}$.

Diseño metodológico. Básico-experimental.

Recolección, selección y secado de la muestra. El propóleo de Apis mellifera fue producido en el distrito de Chalhuanca, a $2897 \mathrm{~m}$ de altura, provincia de Aymaraes, región Apurímac. Se utilizaron dos muestras de propóleo en bruto. El sistema de muestreo fue por conveniencia. Se recolectó de 30 colmenas en las mañanas de clima templado, durante el mes de julio del 2013, mediante raspado directo con espátula de todos los lugares de la colmena en que estuviera depositado, tratando que el producto permanezca en forma de escamas o trozos.

La muestra recolectada se colocó en bolsas plásticas oscuras y luego se procedió a limpiar minuciosamente con ayuda de una pinza para retirar los trozos de madera, astillas de la colmena, abejas muertas, etc. El producto seleccionado se dejó secar alejado de la luz, en habitación ventilada, en recipiente de vidrio, aproximadamente por 15 días, para luego ser conservado en frascos de vidrio color ámbar a temperatura ambiente.

Preparación de la tintura madre de propóleo. El producto, congelado entre $5-10^{\circ} \mathrm{C}$ de 2 a 3 horas para alcanzar consistencia sólida, fue reducido a polvo mediante molienda, para luego ser sometido a maceración en etanol de $96^{\circ}$, en proporción 1:1 peso/volumen, durante 30 días y agitando cada día media hora aproximadamente. Finalmente, previa refrigeración a $8^{\circ} \mathrm{C}$, se filtró en frío para remover las ceras, consiguiéndose así la tintura madre.

Preparación del extracto blando de propóleo. El extracto blando se obtuvo colocando la tintura madre en baño maría a $37^{\circ} \mathrm{C}$ y luego se envasó herméticamente en frasco de vidrio, embalándose adicionalmente con cinta negra aislante para evitar el ingreso de la luz. Se conservó en refrigeración hasta su empleo.

\section{Evaluación de las características organolépticas}

Se realizó en el Laboratorio de Farmacognosia de la Escuela de Formación Profesional de Farmacia y Bioquímica, mediante evaluación sensorial ${ }^{(4)}$.

\section{Determinación de las características fisicoquímicas}

Se realizó siguiendo los procedimientos propuestos por Miranda y Cuellar ${ }^{(4)}$.

Determinación de humedad. Se desecaron $5 \mathrm{~g}$ del extracto a $105^{\circ} \mathrm{C}$ durante 3 horas. Se dejó enfriar 
a temperatura ambiente y se volvió a pesar para nuevamente colocarlo) en la estufa durante 1 hora. Se calculó mediante la siguiente formula:

$$
\% \text { de humedad }=\frac{\text { Pérdida de peso }(\mathrm{g})}{\text { Peso de la muestra }(\mathrm{g})} \times 100
$$

Determinación de cenizas. Se pesaron $3 \mathrm{~g}$ del extracto concentrado en un crisol de porcelana, previamente incinerado y tarado, luego se calentaron suavemente en una cocinilla hasta carbonización, se incineró a $500^{\circ} \mathrm{C}$ en mufla por 3 horas, se enfrió en desecador, se pesó y se calculó mediante la siguiente fórmula:

$$
\% \text { de cenizas }=\frac{M_{2}-M}{\left(M_{2}-M\right) /\left(M_{1}-M\right)} \times 100
$$

Dónde:

$\mathrm{M}$ = Masa del crisol vacío $(\mathrm{g})$

$\mathrm{M}_{1}=$ Masa del crisol con la porción de ensayo $(\mathrm{g})$

$\mathrm{M}_{2}=$ Masa del crisol con la muestra $(\mathrm{g})$

$100=$ Factor matemático para los cálculos

Determinación de sólidos totales. Se colocaron 5,0 $\mathrm{mL}$ del extracto en una cápsula previamente tarada, se evaporó en estufa a $105^{\circ} \mathrm{C}$ hasta peso constante (aprox. 3 horas), se llevó a desecadora hasta que alcanzó la temperatura ambiente, luego se pesó. Se calculó mediante la siguiente fórmula:

$$
\text { St }=\frac{\operatorname{Pr}-\mathrm{P}}{\mathrm{V}} \times 100
$$

Dónde:

$\operatorname{Pr}=$ Masa de la cápsula más el residuo $(\mathrm{g})$

$\mathrm{P}=$ Masa de la cápsula vacía $(\mathrm{g})$

$\mathrm{V}=$ Volumen de la porción de ensayo

$100=$ Factor matemático para el cálculo

\section{Tamizaje fitoquímico}

Las reacciones de identificación de los diferentes metabolitos secundarios del extracto hidroalcohólico se realizaron siguiendo los procedimientos de coloración y precipitación propuestos por Miranda y Cuellar ${ }^{(4)}$.

\section{Determinación de la actividad broncodilatadora}

Se modificó el método de Jagdish et al. ${ }^{(5)}$ para determinar la actividad broncodilatadora, de modo que se indujo la broncoconstricción de los anillos traqueales aislados de cobayos, conservados en el medio nutricio de Tyrode con histamina, y se evaluó el efecto broncodilatador con la sustancia en estudio, utilizando como patrón de referencia salbutamol.

Los animales de experimentación se dividieron aleatoriamente en cinco grupos:

- Grupo I: tratado con $1 \mathrm{~mL}$ de solución de histamina $5 \times 10^{-4} \mathrm{M}$. Blanco.
- Grupo II: tratado con $1 \mathrm{~mL}$ solución de salbutamol $20 \mathrm{mg} / 10 \mathrm{~mL}$. Control.

- Grupo III: recibió $1 \mathrm{~mL}$ del extracto hidroalcohólico de propóleo de Apis mellifera al 5\%.

- Grupo IV: recibió $1 \mathrm{~mL}$ del extracto al 10\%.

- Grupo V: recibió $1 \mathrm{~mL}$ del extracto al 15\%.

Se suspendió la alimentación de los animales 24 horas antes del inicio del procedimiento, siendo mantenidos con agua a voluntad. Luego de calibrar el quimógrafo, se sacrificaron los cobayos por traumatismo encéfalo craneano y se les extrajo la tráquea, colocándola en solución de Tyrode a $37^{\circ} \mathrm{C}$. Luego de lavarlas, se cortó transversalmente entre los cartílagos liberando los anillos traqueales para atarlos con seda quirúrgica por su sección cartilaginosa y muscular, de modo que formen una cadena con su partes musculares alternamente opuestas. Después fueron transferidos a un baño de $25 \mathrm{~mL}$ de solución de Tyrode en burbujeo constante a $37^{\circ} \mathrm{C}$, siendo fijados con seda quirúrgica a un transductor y a la cámara para órganos aislados. Aproximadamente 4 minutos después de encender el software LarChart, se agregó 1 $\mathrm{mL}$ de histamina $5 \times 10^{-4} \mathrm{M}$ y se observó por 4 minutos para el grupo I. Para los demás grupos se hizo un registro de control durante 4 minutos, se agregó 1 $\mathrm{mL}$ de histamina al baño en que estaban inmersos los anillos traqueales y luego de 4 minutos más se adicionó, según correspondiera a cada grupo, $1 \mathrm{~mL}$ de salbutamol o extracto al 5, 10 y $15 \%$, respectivamente, manteniéndose en observación por 20 minutos.

Se hicieron 5 repeticiones por grupo. Todos los cambios y movimientos fueron captados por el transductor y registrados en la computadora.

Para el análisis de datos, se determinó el promedio de las diferencias de tensión generada con histamina, salbutamol y extracto hidroalcohólico de (5, 10 y $15 \%$ ). También se calculó el promedio y los valores de dispersión del número de las contracciones y las alturas alcanzadas (mm) por éstas para cada uno de los tratamientos. Los resultados, presentados comparativamente en tablas y figuras, fueron sometidos al análisis de varianza (ANOVA) que permitió determinar la existencia de diferencias estadísticamente significativas. Las diferencias intergrupos se analizaron por la prueba de Tukey. Para el estudio se utilizó un nivel de confianza $p<0,05$. Se empleó el software estadístico SPSS versión 20,0.

\section{RESULTADOS}

Los resultados se encuentran en las tablas 1-3 y las figuras 1-3. 
Tabla 1. Características organolépticas del propóleo de Apis mellifera recolectado en el distrito de Chalhuanca, provincia de Aymaraes, región Apurímac.

\begin{tabular}{ll} 
Características & \multicolumn{1}{c}{ Descripción } \\
\hline Aspecto externo & Enteras y granos briqueteados \\
Estructura & Heterogénea, presencia de impurezas \\
Color & Pardo oscuro casi negro \\
Olor & Característico, resinoso y aromático \\
Sabor & Amargo picante \\
Consistencia & A temperatura superior a $20^{\circ} \mathrm{C}$, es viscoso, blando y pegajoso, \\
\hline
\end{tabular}

\section{DISCUSIÓN}

El extracto en estudio se preparó con alcohol etílico $96^{\circ}$, por ser éste el más usado y adecuado ya que extrae la mayor cantidad de compuestos químicos presentes en drogas ${ }^{(6)}$.

Según diversos autores, el clima y la ubicación geográfica determinan las propiedades del propóleo bruto, incluyendo sus características organolépticas ${ }^{(7)}$.

Las características fisicoquímicas determinadas para el propóleo (tabla 2) cumplen con la norma vigente del Ministerio de Agricultura del Brasil -humedad $\leq 8 \%$, cenizas totales $5 \%$ y sólidos totales $40 \%-{ }^{(8,9)}$. El bajo porcentaje de humedad hallado en el producto indica mayor concentración de compuestos bioactivos ${ }^{(10)}$.

En el tamizaje fitoquímico se encontró abundante presencia de triterpenos y esteroides, catequinas, resinas, fenoles, taninos y flavonoides, coincidiendo con lo indicado por Chong ${ }^{(11)}$, excepto por la ausencia de cumarinas en la muestra estudiada.

Medina (7), evidenció en propóleo, mediante ensayos cromatográficos CCF y UV-visible, la presencia de flavonoides como crisina, galangina, pinosembrina y apigenina.

Los flavonoides presentes en el propóleo sugieren actividad biológica relacionada con ellos ${ }^{(12)}$. Según Ferré ${ }^{(14)}$, algunos de los componentes fenólicos del propóleo, como ácido caféico, éster del ácido fenil-etilcaféico, quercetina y naringenina, ejercen efectos antiinflamatorios y actúan sobre la producción de eicosanoides, tanto in vitro, suprimiendo la generación de prostaglandinasy de leucotrienos en macrófagos peritoneales, como in vivo, en la inflamación peritoneal aguda inducida por zimosina ${ }^{(13)}$. El propóleo por vía oral suprime significativamente la vía de la lipooxigenasa en el metabolismo del ácido araquidónico, siendo el éster del ácido fenil-etilcaféico el modulador más potente de la cascada del ácido araquidónico. Tanto el propóleo como los flavonoides disminuyen la

\section{losdañosproducidosporlainterleuquina-13 (13) Sesabeque}

Tabla 2. Características fisicoquímicas del propóleo.

\begin{tabular}{lc} 
Características & Resultados (\%) \\
\hline Humedad & 4,264 \\
Cenizas totales & 1,290 \\
Sólidos totales & 17,188 \\
\hline
\end{tabular}

actividad de la ciclooxigenasa en macrófagos -medida en función de la producción de prostaglandina E2- y protegen al tejido cartilaginoso y a los condrocitos humanos de losdaños producidosporlainterleuquina-1 $ß^{(13)}$. Sesabeque la miricetina y quercetina, a concentraciones relativamente altas in vitro, bloquean la acción de la ciclooxigenasa y la lipoxigenasa, mientras a concentraciones bajas inhiben sólo a esta última. Estas propiedades antiinflamatorias también se han demostrado in vivo para numerosos flavonoides en distintos modelos de inflamación aguda o crónica. Entre estos cabe mencionar: nepetina, apigenina, rutina, crisina, quercetina, quercitrina y luteolina ${ }^{(14)}$.

De esto se infiere que el propóleo no tiene un mecanismo broncodilatador específico siendo los flavonoides y compuestos fenólicos quienes le confieren propiedades terapéuticas.

El ensayo farmacológico se realizó teniendo en cuenta que los anillos traqueales aislados de cobayo mantienen su función decontraccióny relajación cuando se encuentran inmersos en soluciones adecuadas, Jagdish et al. ${ }^{(5)}$, por tal motivo se mantuvieron inmersos en solución de Tyrode a $37^{\circ} \mathrm{C}$ con adecuada oxigenación constante desde su aislamiento. Los resultados fueron recogidos por el software LabChart que registra la tensión mecánica de la contracción y relajación del órgano aislado para convertirla en señal eléctrica.

En la figura 1, se observa que el extracto hidroalcohólico de propóleo $5 \%$, redujo a 10,818 g

Tabla 3. Metabolitos secundarios presentes en el extracto hidroalcohólico del propóleo.

\begin{tabular}{|c|c|c|c|}
\hline $\begin{array}{l}\text { Metabolitos } \\
\text { secundarios } \\
\end{array}$ & Ensayo & Resultados & Observación \\
\hline $\begin{array}{l}\text { Triterpenos y/o } \\
\text { esteroides }\end{array}$ & Lieberman-Burchard & +++ & $\begin{array}{l}\text { Rosado a } \\
\quad \text { rojo }\end{array}$ \\
\hline Catequinas & Catequinas & +++ & $\begin{array}{l}\text { Verde } \\
\text { carmelita }\end{array}$ \\
\hline Resinas & Resinas & +++ & Precipitado \\
\hline Azúcares reductores & Benedict & ++ & $\begin{array}{l}\text { Precipitado } \\
\text { rojo }\end{array}$ \\
\hline Fenoles y/o Taninos & Cloruro férrico & +++ & $\begin{array}{l}\text { Verde } \\
\text { azulado }\end{array}$ \\
\hline Flavonoides & Shinoda & +++ & Rojo \\
\hline Flavonoides & Antocianidinas & +++ & $\begin{array}{l}\text { Rojo a } \\
\text { marrón }\end{array}$ \\
\hline
\end{tabular}

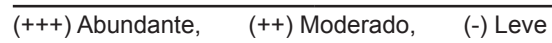




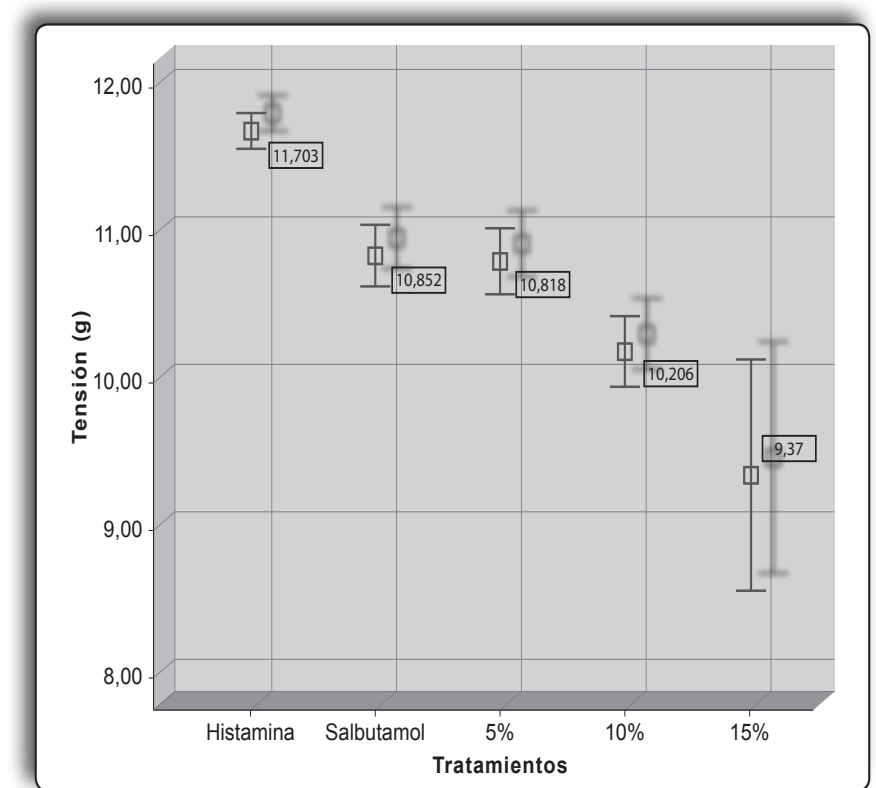

Figura 1. Tensión (g) de la contracción de los anillos traqueales según tratamientos.

la tensión generada por histamina; efecto similar al salbutamol (10,852 g). Las concentraciones de $10 \mathrm{y}$ $15 \%$ redujeron aún más la tensión llegando a 10,206 y 9,371 g, respectivamente. Se estableció así una relación directa dosis-respuesta, puesto que a mayor dosis se obtuvo mayor disminución de la tensión generada.

La figura 2 muestra el número de contracciones generada por cada tratamiento. Salbutamol disminuye el número de contracciones generadas por la histamina a 21,40, seguido por el extracto de propóleo al 5\% con 19,20 , mientras que a concentraciones 10 y $15 \%$ se obtuvieron 15,40 y 9,80 ; respectivamente.

La figura 3 muestra, que la altura de las contracciones son menores respecto de la alcanzada con histamina.

Para estos tres parámetros, la prueba de Tukey mostró que el extracto al $5 \%$ y salbutamol tienen efectos similares.

El análisis de varianza para el grado de tensión, el número y altura de contracciones, muestra que existen diferencias significativas en los diferentes tratamientos, evidenciando que las tres concentraciones trabajadas tienen efecto broncodilatador.

Otras especies también tienen potencial broncodilatador. Según Bautista ${ }^{(15)}$, el extracto fluido de Mentha arvensis L. "hierba buena" al 10 y $15 \%$, presenta este efecto en traquea aislada de cobayo con valores de disminución estadísticamente similares a los obtenidos aquí con propóleo, mientras que al $20 \%$ presenta una disminución de la respuesta contráctil estadísticamente similar al salbutamol $2 \mathrm{mg} / 5 \mathrm{ml}(p>0,05)^{(15,16)}$.

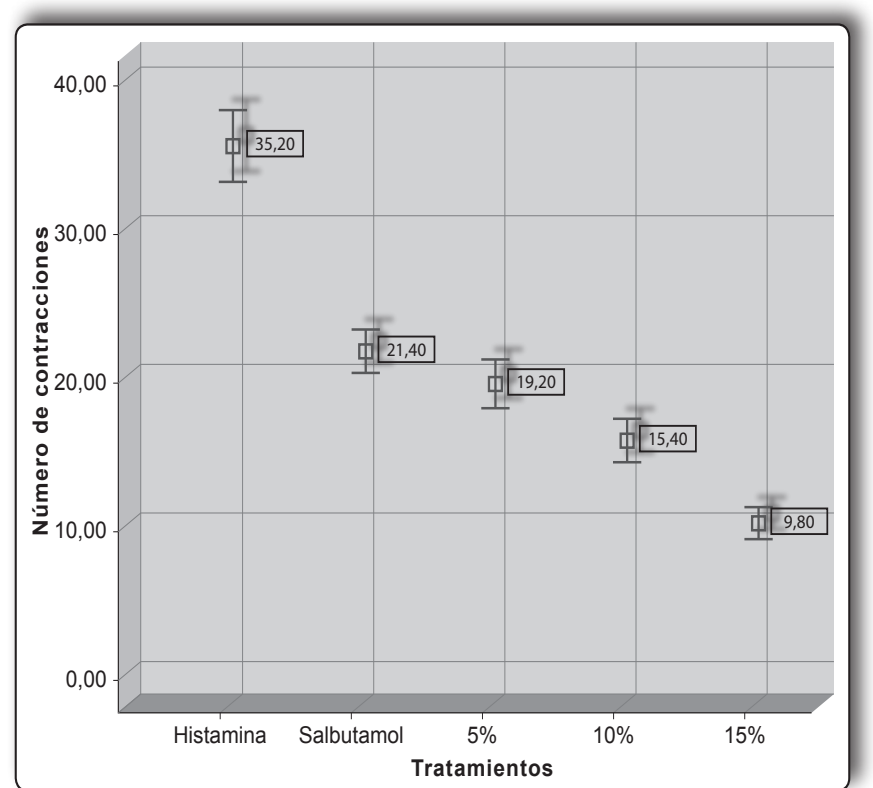

Figura 2. Número de contracciones de los anillos traqueales en respuesta a los tratamientos.

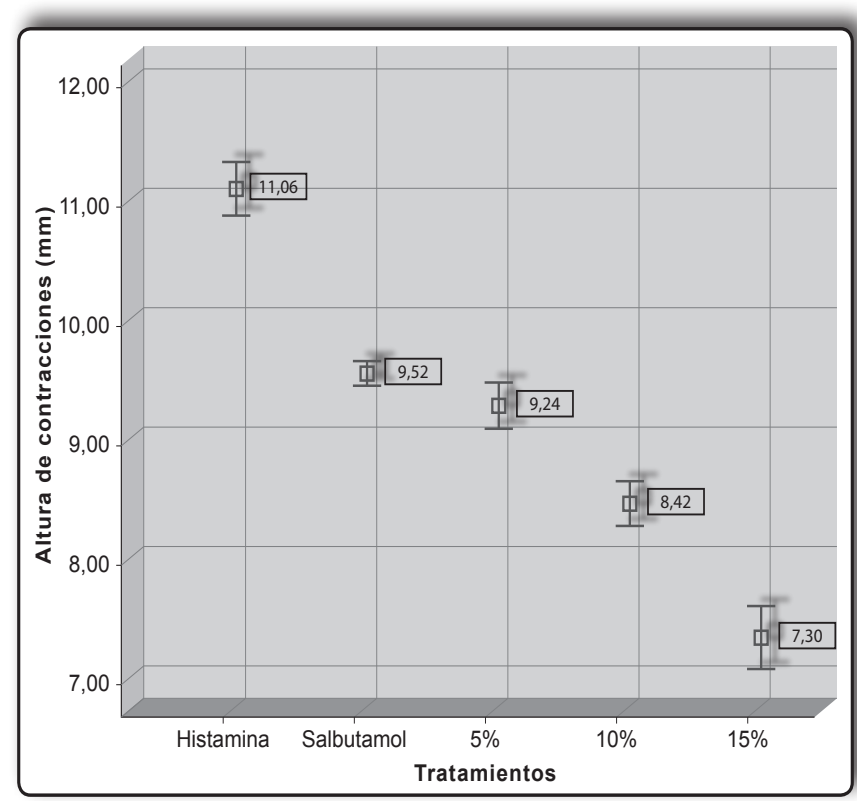

Figura 3. Altura de las contracciones de los anillos traqueales en respuesta a los tratamientos.

\section{CONCLUSIONES}

A condiciones experimentales, se demostró que la actividad broncodilatadora del extracto de propóleo al $5 \%$ es estadísticamente similar a la del salbutamol (20mg/10ml), y que a la concentración $15 \%$ disminuye aún más el número de contracciones (figura 2).

También se observa un inconveniente, conforme pasa el tiempo, disminuye el número y la altura de contracciones; esto podría deberse a la falta de afinidad 
con los receptores histaminérgicos o a que se utiliza otro tipo de receptores.

En el estudio in vitro se encontró que el 1,8-cineol tiene efectos farmacológicamente importantes, relaja los músculos lisos de la tráquea de cobayos manteniéndolos en tono basal, y disminuye los marcadores de procesos inflamatorios en las vías respiratorias.

\section{REFERENCIAS BIBLIOGRÁFICAS}

1. Organización Mundial de la Salud [OMS]. Enfermedades respiratorias Crónicas. Journal of asthma [Internet]. 2011 [Citado 20 oct 2013]: 52(8). Disponible en http://www. who.int/features/factfiles/asthma/es/index.html

2. Castaldo S, Capasso F. Propolis, an old remedy used in modern medicine. Fitoterapia [Internet]. 2002 [Citado 22 set 2013]; 73 Suppl 1: S1-6. Disponible en: http://www. ncbi.nlm.nih.gov/pubmed/12495704

3. Gómez-Caravaca A, Gómez-Romero M, Arráez-Roman D, Segura-Carretero A, Fernández-Gutierrez A. Advances in the analysis of phenolic compounds in products derived from bees. Journal of Pharmaceutical and Biomedical Analysis [Internet]. 2006 [Citado, 22 set 2013]; 41(4): 1220-34. Disponible en: http://www.scienced rect.com/ science/article/pii/So731708506002536

4. Miranda M, Cuellar A. Manual de Prácticas de Laboratorio: Farmacognosia y Productos Naturales. La Habana, Instituto de Farmacia y Alimentos. Universidad La Habana. Cuba. 2000.

5. Jagdish B, Sandip A. Antiasthmatic Activity of Leptadenia reticulata (Retz) Wt\& Arn leaves. Procedente de Planetary Scientific Research Center Conference Proceedings Volume 31; 2013 abr 29; Singapur. [Citado 14 ago 2013]. Disponible en: http://cstm.cnki.net/stmt/TitleBrowse/ Detail?baseid=PSRC201304002

6. Luna L. Estudio del efecto de dos promotores inmunológicos de origen natural (propóleo, polen) y su incidencia en la producción de pollos de engorde, en el sector El Tejar, provincia de Imbabura. [Tesis de Pregrado]. Pontificia Universidad Católica de Ecuador sede Ibarra. Ibarra, 2011.

7. Medina A. Flavonoides aislados de propóleos chilenos y bioactividad. [Tesis de pregrado]. Universidad Austral de Chile. Valdivia, 2012.

8. Reyes C. Actividad antibacteriana in vitro del extracto etanólico de propóleo peruano sobre cultivos de bacterias anaerobias frecuentes en pacientes con periodontitis crónica. [Tesis Pregrado] Universidad Nacional Mayor de San Marcos. Lima, 2010.

9. Instrução Normativa № 3 - ANEXO VI. Reglamento técnico para fixação de identidade equalidade de própolis. Ministerio de Agricultura del Brasil. Diario Oficial da República Federativa do Brasil, 19 jan 2001. [Citado 18 nov 2013]. Disponible en http://www.apacame.org.br/ mensagemdoce/6o/normas.htm

10. Martínez G. Caracterización físico - química y evaluación de la actividad antifúngica de propóleos recolectados en el suroeste antioqueño. [Tesis de Maestría]. Universidad de Colombia sede en Medellín. Medellín, 2009.

11. Chong A. Formulación y evaluación de la actividad antitusígena del jarabe elaborado a base de extracto hidroalcohólico de propóleo de Apis mellifera "abeja". [Tesis Pregrado]. UNSCH. Ayacucho, 2008.

12. Ángel C. Evaluación de la actividad genotóxica de propóleos recolectados en diferentes apiarios de Cundinamarca y Boyacá. [Tesis de maestría]. Universidad Nacional de Colombia. Bogotá, 2012.

13. Farré R. Frasquet I. Sánchez A. El própolis y la salud. Ars Pharmaceutica. [Internet] 2004 [Citado 18 nov 2013] 45(1): 21-43. Disponible en http://digibug. ugr.es/bitstream/10481/28176/1/Ars\%2oPharm\%20 $2004 \% 3{ }_{45}(1) 21-43 . p d f$

14. Bonkanka T. Evolución farmacológica de terpenos y flavonoides de origen vegetal. [Tesis de doctorado]. Universidad de la Laguna. Tenerife, 2007.

15. Bautista L. Evaluación del efecto broncodilatador del extracto fluido de Mentha aff. arvensis L. "hierba buena" en tráquea aislada de Cavia porcellus "cobayo". [Tesis Pregrado]. UNSCH. Ayacucho, 2012.

16. Rathore C, Dutt KR, Sahu S, Deb L) Antiasthmatic activity of the methanolic extract of Physalis angulata Linn. J Med Plants Res. [Internet]. 2011 [Citado 18 jul 2013]; 5(22): 5351-5. Disponible en: http://www.academicjournals. org/journal/JMPR/article-full-text-pdf/5B1682C1635o)

Manuscrito recibido el: 09/o6/2015

Aceptado para su publicación el: 20/04/2016

\section{Correspondencia:}

Nombre: $\quad$ Mario Carhuapoma Yance

Dirección: Jr. Puno 1002 - Lima o1

e-mail: mariocarhuapomayance@hotmail.com 